\title{
Report Stating the Concept of Cloud Computing Testing and Its Challenges
}

\author{
Amit Saxena \\ Research Scholar, \\ AGSER, India, \\ amit.saxena088@gmail.com
}

Abstract: - These days Cloud Computing is in demand and the latest technology used by the organisation to handle large amount of data. This paper will explain the concept of Cloud Computing, its models, advantages, disadvantages and also the challenges. The type of testing use for cloud computing will also be discussed in this paper. The organisations may have their own cloud data centre or also can tie up with other data service providers who provides the facility of cloud computing. The data is shared among various servers and then can be accessed from there. It helps to increase the efficiency of the business organisation.

Keywords: - Cloud Computing, Testing, Advantages, Disadvantages, Challenges.

Introduction: - The process of providing data centres to the customers over the internet is called as cloud computing. This is in trend these days and have many benefits. Some organisations have their own cloud to have all the data collected at same place. But in some organisations in order to save time to maintain the data clouds, they rent it from outside providers. In this case the cost factor is high but most of the companies are doing this way. They either hire from outside resources or rent the facility. There are following models of the cloud computing technology: -

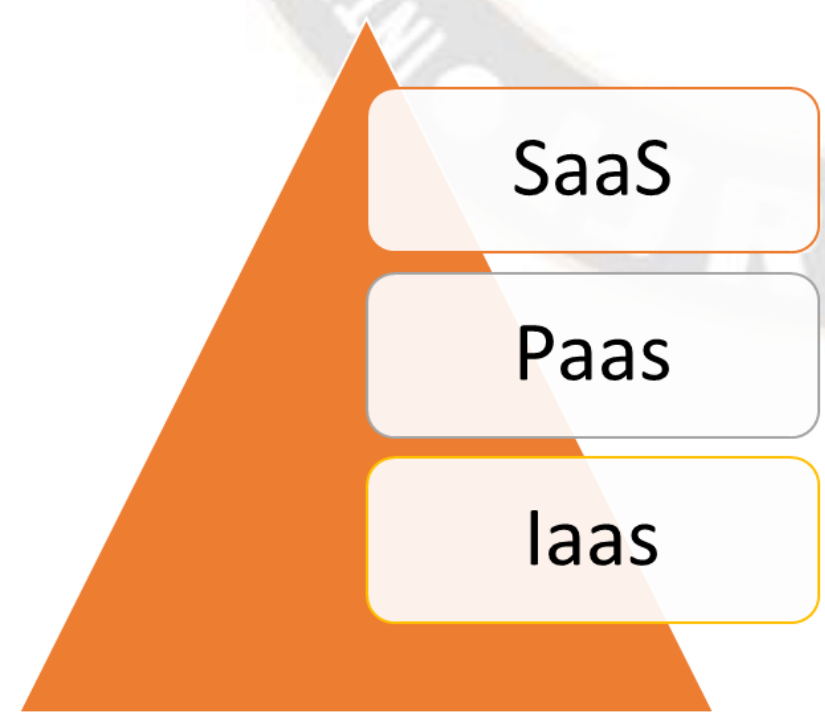

Figure 1 Cloud Computing Model
There are three ways that the cloud computing providers provide their services. They provide services as infrastructure, platform or software services. They all are independent of each other. This is the benefit of these types of service providers that they can also provide only one type of services to their clients based upon the client's needs. Following are the details of each type of services provided: -

1. SaaS (Software as a Service): - In this type of service model, the providers gives the option to use various software applications which are available on centralised cloud to their users. In this case the users are not supposed to manage the infrastructure, database etc but all that is managed and maintained by the providers. The users can use all the application services provided by software-based cloud.

2. PaaS (Platform as a service): - In this type of model, the provider provides a platform to the developers to develop the applications. So, the cloud providers will provide development platforms which can include all the things available at one place like operating system, data base, web server etc. In this way the developers can develop their applications and can run on these provided platforms by the cloud providers without even having the platform in their own organisation. This way they can save a lot of cost.

3. IaaS (Infrastructure as a Service): - In this type of model, the computer infrastructure is presented in the 
form of virtualisation. All the services related to infrastructure is provided to the end user virtually. The user does not control the infrastructure but can use all the services like storage, operating system, etc.

\section{Cloud Computing Testing Methodology: - [1]}

Cloud Testing is the process of testing the cloud services, environment, web etc using cloud- based testing tools. The Cloud testing focuses on many factors like functional and non-functional parameters. It is the process of identifying all the issues that might occur in a cloud-based system. The cloud in fact provides variety of tools to do the testing of the services, infrastructure and platform of the clouds. There are following three types of testing in clouds: -

\section{Testing \\ within a}

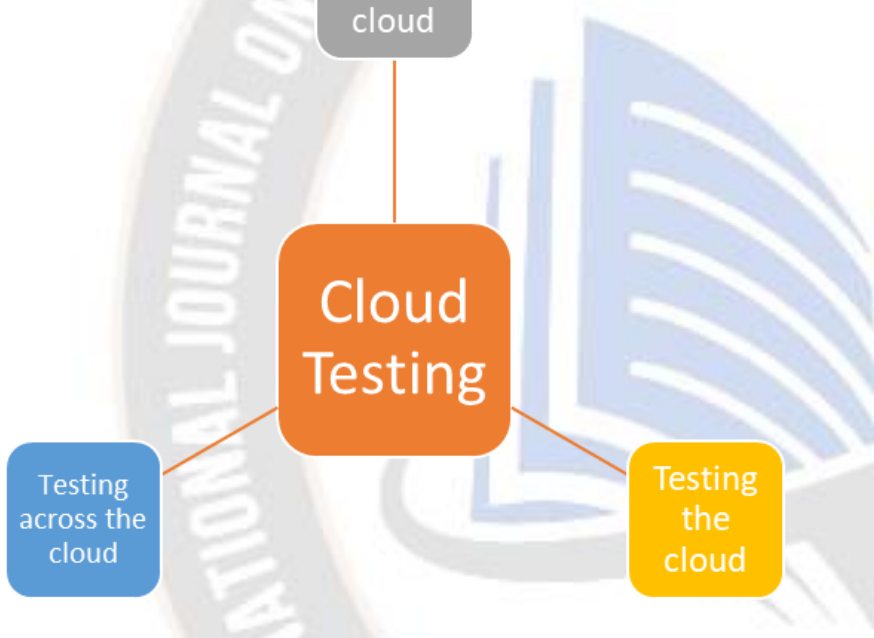

Figure 2 Cloud Testing Methodology

a. Testing within a Cloud: - This type of testing means testing all the services provided by that particular cloud. This type of testing is done by the provider of the cloud. Before providing services to the client via cloud, he must make sure that the services are working without any errors and can be used to increase the efficiency of the organisation using it. Hence, the cloud provider must follow all the testing processes to test the cloud.

b. Testing the Cloud: - Based on the attributes and characteristics of cloud the testing is done. This type of testing is done by the users as well as the SaaS cloud providers. This way the user can also test the cloud before they make a deal with the providers. If they find any errors then they can ask the providers to fix the errors before they start using the technology. c. Testing across the Cloud: - This type of testing includes testing of different types of clouds like public, private or hybrid cloud.

Importance of Cloud Testing: - The concept of cloud technology is new and very useful and similar way it is very important to test the cloud before it is given to the end user. There are certain services which are centralised and whose testing is very important to do so that when the user starts using them, they should not find any error or difficulty using them. There are many factors involved like type of services provided, their compatibility with various platforms, type of browsers used etc. Hence, this all should be tested before the end user starts using them.

Cloud Testing Technique: - The testing life cycle of cloud is similar to the software testing life cycle. It has various stages which should be followed in order to make sure that the cloud is tested properly and have no issues: -

a. Information Gathering: - In this stage first of all the testers will sit with the provider and try to understand the type of services provided by the cloud. They will identify the type of browsers used, platform, operating system etc in this phase and prepare the document which will have detailed information about the services provided by that particular cloud.

b. Build Test Cases: - Based upon the document of the information gathering stage, the testers start preparing the test cases. They will consider various factors which are type of services provided, which platform will be used, operating system. They will also make test cases to test the network and servers which are used by this particular cloud.

c. Run the test cases: - Once the test cases are built then in this phase the tester starts executing all the test cases and observe if there are any bugs. All the test cases are executed in this phase.

d. Fix The issues: - In this phase based on the bugs report the bugs are being fixed.

e. Deployment: - In this phase, once there are no bugs or errors remaining, finally the cloud is deployed for the user to start using it. 


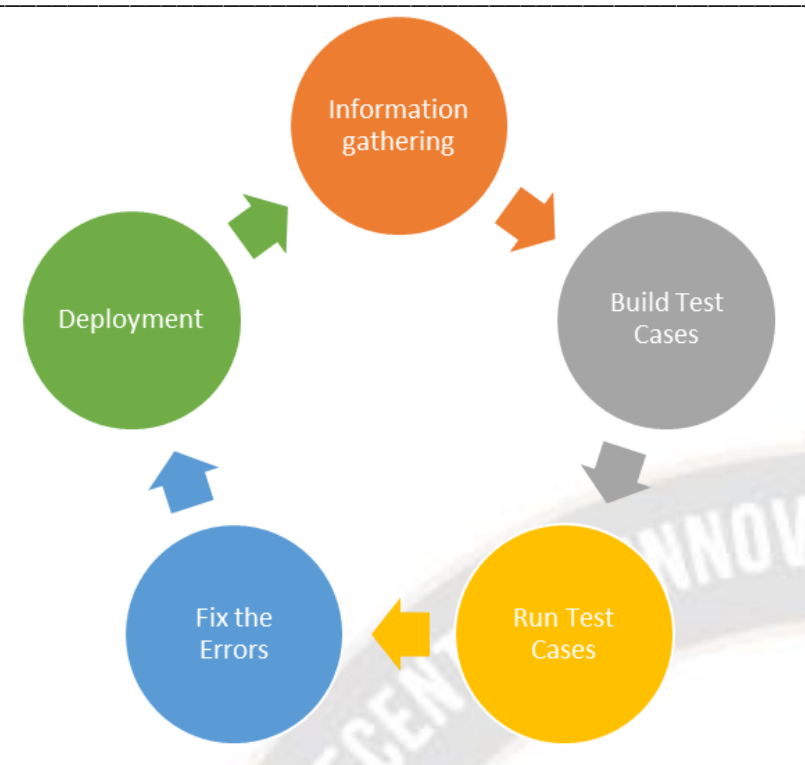

Figure 3 Cloud Testing Life Cycle

Advantages of Cloud Testing: - [2] The benefit of cloud testing is that the cloud itself provides many tools to test the cloud. Hence there are many benefits of the cloud testing: -

a. Reduces Cost: - As the cloud provider provides tools for testing the cloud, the cost factor reduces to a large extent. There is not need to buy new tools from outside and the tester can start testing the services provided by the cloud using the tools available in the market.

b. Reduced time factor: - As the tools are automated so a lot of time is saved while testing the services of the cloud.

c. Non stop availability: - As all the services provided by the cloud is available all the time provided that the user has good internet connectivity, it is very easy for the testers to test the services or software at any given point of time.

d. Can be customised: - Based on the type of software to be tested the testers can customise the type of tools needed to perform their testing.

Challenges of Cloud Testing: - [3]

There are many challenges related to testing of cloud services. The testing is important but it has its own challenges which are discussed in following points: -

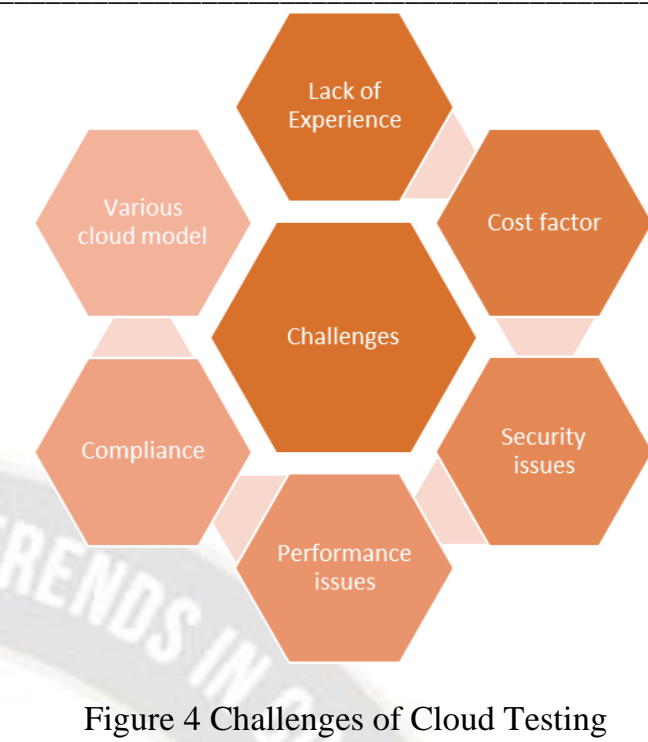

1. Lack of Experience: - This is the main challenge while testing the cloud. If the tester is not knowledgeable enough about the techniques then he will not be able to do proper testing of the cloud. Also, if there are less resources it will be very difficult for the organisations to maintain the technology.

2. Cost Factor: - Not all organisations are capable of putting a lot of cost in order to use the testing tools provided by the provider. Sometimes if the provider provides services which are less in cost but not good in quality then also the purpose of business having cloud will not be met.

3. Security Issues: - What ever is available online will always have the lack of security issues. The private information can be leaked. This is major challenge of the cloud testing. Even if the tester is efficient sometime it is very difficult to identify all the threats online.

4. Performance Issues:-In some situations the services provided by the vendors are not of good quality and in some case, there are chances of failure of the whole concept of the cloud. Hence, the end users will never trust such vendors and the services provided by them. And also, there is loss for the vendors.

5. Compliance: - This is about following all government rules and regulations which are given by the government for the safety of the confidential information. In some cases, the vendors are not able to met all these policies which in turn will result in loss of trust in vendor services provided to the users and there will be less users attracted towards such vendors. 
6. Various Cloud Models: -Some business uses more than one cloud service by various vendors which in turn make it more challenging to manage all the information as well as the privacy of the confidential information.

Types of Testing in Cloud Computing: -[4]

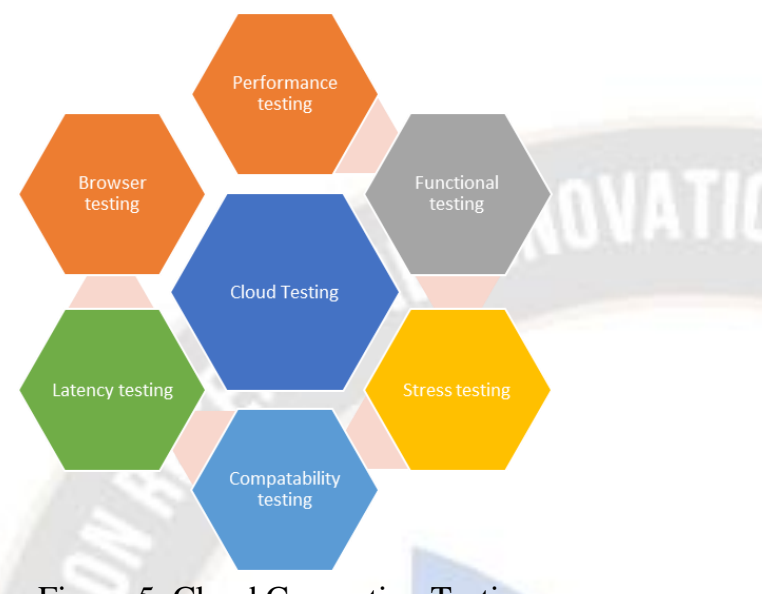

Figure 5. Cloud Computing Testing

a. Performance testing: - Performance testing means testing the overall performance of the services provided by the vendors. This is to test how the services will react in case of heavy load and under increased number of users. It is to test whether the cloud is able to perform under heavy load conditions or it fails under such conditions. This also makes sure that the services works at same efficiency even when one uses different types of devices. Even if the same cloud is provided to different businesses then also the vendor should make sure that the cloud services will work normally without any interruptions.

b. Stress Testing: - In this type of testing it is made sure that the applications work same under different load conditions. This is to test how the system will behave if the load is increased under various conditions over a specific period of time. In this it is also identified if there are any environmental failures in future. It means that the compatibility of the services provided by that particular cloud is with all the platforms used by the end user only then this particular cloud is being used by the end user.

c. Functional Testing: - In this type of testing the tester will test whether the supposed functions to be performed by the services are being performed or not. Which means the testers will test that all the functions of the cloud can be accessed by the end user without any error and without any interruptions. It is tested whether by giving certain input expected output is the result or not. For example, if the customer login then the site should show his login details. d. Compatibility testing: - In this type of testing the testers check the compatibility of the services provided by the cloud. This is to test whether it will be compatible with the end user environment or not. In this testing the testers will also test are the users able to connect to the cloud or not. It is to resolve the login issues. The testers will test whether all the services are compatible on various platforms.

e. Latency Testing: - In this type of testing the testers will test whether they are getting certain response after a particular action is executed or not.

f. Browser Testing: - In this the compatibility of the services by the cloud is checked across all the available browsers.

g. Network Testing: - This type of testing is also important as the security of the applications on cloud are very important. The testers will test the connectivity and also the protocols of the network. The data integrity is also tested while transmission of data.

h. Security testing: - The privacy of the data should be maintained. The testers make sure that only authorised users should get the access to the services on the cloud. The anti-virus, firewall etc is also tested.

Tools Used for Cloud Testing: - [5]

There are many tools available for cloud testing: -

*LoadStorm

*Blazemeter

*Nessus

*App Thwack

Conclusion: - Cloud computing is very much use in these days and it is mostly used by all the organisations. There are various types of services provided by the cloud vendors. Each type of services provided by the cloud has its own advantages and disadvantages. But it is observed in this paper that the advantages are more and it is very convenient way of using the services without implementing it in own business. In fact, the concept also provides various testing tools to the testers with the help of which the work of testers become easy.

\section{References: -}

1.https://www.cigniti.com/blog/top-7-cloud-based-softwaretesting-tools/

2.https://dzone.com/articles/what-are-the-primary-benefits-ofcloud-testing

3https://medium.com/@ImpactQA/7-major-challenges-oftesting-in-a-cloud-computing-environment-da766fd980ec

4.https://www.guru99.com/cloud-testing-tutorial-with-saastesting-primer.html 